\title{
Intertrial priming of pop-out search influences the shift, skew, and dispersion of response time distributions
}

\author{
Bryan R. Burnham ${ }^{1} \cdot$ James J. Cilento $^{1} \cdot$ Bridget Hanley $^{1}$
}

Published online: 21 April 2015

(C) The Psychonomic Society, Inc. 2015

\begin{abstract}
Priming of pop-out ( $\mathrm{PoP})$, or intertrial priming, is the finding that responding to a singleton target is faster when a target's defining feature (e.g., color) and nontarget features are repeated between trials than when the target and nontarget features switch between trials. Facilitated responding may reflect priming's influence on selection, that is, implicitly encoded features speed the selection of a matching target. In contrast, PoP effects may also reflect intertrial priming's influence on postselection processes, where episodic retrieval of a previous target is facilitated when its features match the current target. Lamy, Yashar, and Ruderman Attention, Perception, \& Psychophysics, 73, 2160-2167 (2011) proposed a hybrid, dual-stage model that assumes intertrial priming influences both selection and postselection retrieval. To provide support for intertrial priming influencing more than one cognitive process, we examined priming's influence on the shift, skew, and dispersion of RT distributions in PoP tasks by fitting the exponential-Gaussian function to the RTs. Three experiments demonstrated that PoP effects at the level of mean RT were associated with changes in both the shift and skew of the underlying RT distributions. Importantly, Experiments 2 and 3 showed that manipulations intended to influence selection or postselection processes produced corresponding changes in the contributions of the distribution shift and skew to the PoP effects on mean RT. The results suggest more than one process is influenced by intertrial priming in visual search
\end{abstract}

Bryan R. Burnham

attention.perform@gmail.com

1 Department of Psychology, University of Scranton, Scranton, PA 18510, USA tasks, but readers should be cautious about relating specific processes to specific exponential-Gaussian parameters.

Keywords Priming of pop-out · Visual search · Priming · Attention response time distributions

A ubiquitous finding in the visual search literature is that visual search is facilitated when target and distractor features are repeated across trials (e.g., Chun \& Jiang, 1998; Chun \& Nakayama, 2000; Lamy, Antebi, Aviani, \& Carmel, 2008; Lamy, Yashar, \& Ruderman, 2010; Maljkovic \& Nakayama, 1994, 1996, 2000; Müller, Heller, \& Ziegler, 1995; Wolfe, Butcher, Lee, \& Hyle, 2003). For example, Maljkovic and Nakayama (1994) demonstrated that during search for a singleton target (pop-out search), when there was uncertainty about whether the target would be a red item among green items or a green item among red items, repeating the target color sped responding, relative to when the target and distractor colors "switched" across trials. This priming of pop-out ( $\mathrm{PoP})$ or intertrial priming effect has been observed with color (Goolsby \& Suzuki, 2001; Olivers \& Humphreys, 2003), orientation (Hillstrom, 2000; Olivers \& Humphreys, 2003), and shape (Lamy, Carmel, Egeth, \& Leber, 2006). Intertrial priming effects have also been found with size (Huang, Holcombe, \& Pashler, 2004) and when targets are defined by a conjunction of features (Kristjánssona, Wang, \& Nakayama, 2002), and the results are generally the same: RTs are faster and accuracy is greater when the target features and distractor features repeat than when those features switch or are not repeated. Such results suggest that features orthogonal to a task are implicitly encoded into short-term memory and facilitate search. 
According to selection accounts (e.g., Chun \& Nakayama, 2000; Goolsby \& Suzuki, 2001; Maljkovic \& Nakayama, 1994, 1996), the repetition of target and distractor features speeds the selection of those same features on the following trial. When the target and distractor features from trial $n-1$ repeat, this facilitates selection of and responding to the target on trial $n$, but impedes selection when the target and distractor features switch roles across trials $n-1$ and $n$, by allocating attention toward the distractors. In line with selection accounts, Maljkovic and Nakayama $(1994,1996)$ found that repetition of noncritical features such as the target's response feature did not influence PoP effects. Because response features would exert their influence only after a target is selected and a response is prepared, this suggested that priming influences processes prior to response execution. Similarly, Bichot and Schall (2002) found that neural activation in areas of frontal eye field in macaque monkeys was greater on repeated-target trials than on switch trials, suggesting that early selection processes underlie intertrial priming. Becker (2008) found that target repetition sped saccades to a target's position, suggesting that target repetition influences processes prior to the selection and execution of a response suggests that intertrial priming effects manifest during early selection processes.

In contrast, according to retrieval accounts (e.g., Hillstrom, 2000; Huang et al. 2004; Huang \& Pashler, 2005; Thomson \& Milliken, 2011, 2012, 2013), intertrial priming exerts an influence only after a target has been selected, but before responding. Specifically, after a target has been selected, the visual system verifies whether this item is the target by comparing its features to those of recently encountered targets, such as the target on trial $n-1$, and this episodic retrieval is facilitated if the target features on trial $n-1$ are repeated. Indeed, Thomson and Milliken $(2012,2013)$ demonstrated that PoP effects lasted for lags of 16 trials, suggesting that memory of previous targets was retrieved and facilitated responding. Additional support for retrieval accounts has come from Huang et al. (2004), who found that repetition of the target feature interacted with repetition of the response: Target feature repetition facilitated target search only when the previous response was also repeated. Because activation of a target's response occurs only after a target is selected, this suggests that target feature repetition influenced postselection processes. Additionally, Thomson and Milliken (2011) had subjects complete letter identification and orientation judgment tasks, with these tasks switching in a predictable manner across trials, and found that task repetition resulted in larger intertrial priming effects than when tasks switched. Because the influence of the trial $n-1$ task can influence trial $n$ only after a target is selected, these results suggest that intertrial priming influenced postselection processes.

Selection accounts and retrieval accounts represent the ends of a continuum of explanations for intertrial priming. Indeed, in a review of the repetition-priming literature,
Kristjánsson and Campana (2010) concluded that no one cognitive process can explain all of the empirical findings that show an influence of intertrial priming on search. In that case, what processes might be influenced by intertrial priming?

Lamy et al. (2010) (Yashar \& Lamy, 2011) proposed a dual-stage account that proposes how intertrial priming influences selection and postselection retrieval processes. Lamy et al. (2010) examined the time course of the interaction between response repetition and PoP effects, assuming the influence of response repetition can manifest only after target selection. On some trials, a color change occurred to the items in the search display; that is, a display might initially contain a red target and green distractors, but after 100,200 , or $400 \mathrm{~ms}$, the target color could change to yellow and the distractors to blue. If intertrial priming influenced selection, response repetition should not influence PoP when the color change occurred soon after the display's onset $(100 \mathrm{~ms})$; and if intertrial priming influenced postselection retrieval, response repetition should influence PoP when the color change occurred 200$400 \mathrm{~ms}$ after display onset, because there would be sufficient time to compare the initial target color to the preceding target. As predicted, Lamy et al. (2010) found that PoP effects were unaffected by response repetition when the color change occurred $100 \mathrm{~ms}$ after display onset, but $\mathrm{PoP}$ did interact with response repetition when the color change occurred 200 or $400 \mathrm{~ms}$ after display onset. Taken together, the results suggest that intertrial priming influences both selection and postselection processes.

Recently, we (Burnham, 2013) combined a PoP task with a go/no-go task in which a display feature indicated whether a subject was to respond on each trial. In two experiments, we found that the go/no-go task modulated intertrial priming. In our Experiment 1, the distractor color was the go/no-go feature, and subjects did not respond when distractors were a specific color but did respond otherwise. We found that responding was slower when the trial $n$ target was the same color that the "no-go distractors" had been on trial $n-1$ than when trial $n-1$ had been a go trial. This suggests that the trial $n-1$ distractor color was retrieved after selecting the trial $n$ target, slowing responding when that color was associated with "do not respond." Importantly, responding was slower following go than following no-go trials when the trial $n$ distractor was the same color as the trial $n-1$ target. This suggests that on go trials the target color was encoded and had a biasing effect toward distractors of the same color on trialn, but this effect was less pronounced following no-go trials, suggesting that intertrial priming was influencing selection. Taken together, the results suggest that intertrial priming influences both selection and retrieval.

The studies described above, among others, have shown that experimental manipulations can moderate the size of 
intertrial priming effects at the level of mean response time $\left(M_{\mathrm{RT}}\right)$, which can provide evidence of what cognitive processes underlie intertrial priming effects. However, one remaining question is what influence intertrial priming has on the shift, skew, and spread of the underlying RT distributions. It is well established that RT distributions tend to be positively skewed, and because skew influences the mean, any difference in $M_{\mathrm{RT}}$ between conditions may not reflect a shift in the central tendency of the distributions, but rather a change in skew (e.g., Andrews \& Heathcote, 2001; Balota \& Yap, 2011; Balota, Yap, Cortese, \& Watson, 2008; Heathcote, Popiel, \& Mewhort, 1991; Luce, 1986; Van Zandt, 2000; Yap, Balota, Cortese, \& Watson, 2006). As such, various analytic methods have been used to examine how manipulations that influence $M_{\mathrm{RT}}$ do so by influencing the shift of an RT distribution, its skew, or both.

One method would be to fit mathematical functions to RTs and extract parameter estimates of the central tendency, dispersion, and skew of the RT distribution. One function is the exponential-Gaussian (ex-Gaussian), which is a convolution of a Gaussian (normal) distribution and an exponential distribution (Fig. 1). Others include the Weibull, gamma, and exWald, which has been used to model RTs in simple choiceresponse tasks (Heathcote, 2004; Schwarz, 2001). In the present study, we focused on the ex-Gaussian for three main reasons: First, Palmer, Horowitz, Torralba, and Wolfe (2011) found that the ex-Gaussian was the best-fitting function for the RTs obtained in visual search tasks, which made the exGaussian a better choice for modeling RTs obtained in pop-out visual search tasks. Second, studies on semantic priming have used the ex-Gaussian to model RTs from those tasks and, given that the present study examined priming, this made the ex-Gaussian a better choice (e.g., Balota et al. 2008; Yap et al. 2006). Lastly, the ex-Gaussian has been used to help infer cognitive processes, a point to which we will return below and in the General Discussion. For these reasons, we fit the ex-Gaussian function to the RTs collected in three versions of a PoP task, in order to examine how priming influenced the shift, skew, and dispersion of the underlying RT distributions.

The ex-Gaussian is defined by three parameters (Fig. 1): $\mu$, the mean of the Gaussian portion of the distribution; $\sigma$, the standard deviation of the Gaussian portion; and $\tau$, which is the central tendency and variance of the exponential portion and estimates a distribution's positive skew. The sum of $\mu$ and $\tau$ is equal to the mean of the ex-Gaussian; hence, $M_{\mathrm{RT}}=\mu+\tau$, with $V A R=\sigma^{2}+\tau^{2}$ (Balota \& Spieler, 1999; Balota \& Yap, 2011; Heathcote, Brown, \& Mewhort, 2002; Heathcote et al. 1991; Hockley, 1984; Luce, 1986; Ratcliff, 1978; Van Zandt, 2000). What makes the ex-Gaussian appealing is its usefulness for showing how differences in $M_{\mathrm{RT}}$ reflect changes in $\mu$ (shift), $\tau$ (skew), or both $\mu$ and $\tau$. For example, Balota and Spieler (1999) found that lexical decision times (in $M_{\mathrm{RT}}$ ) were less for high-frequency words than for low-frequency words, and that the differences in $M_{\mathrm{RT}}$ reflected changes in $\mu$ and $\tau$, where $\mu$ was smaller for high-frequency words and $\tau$ was larger for low-frequency words. Additionally, Balota et al. (2008) found that semantic-priming effects on $M_{\mathrm{RT}}$ reflected changes in only $\mu$, but that the increase in semantic priming in $M_{\mathrm{RT}}$ for perceptually degraded targets reflected changes only in $\tau$. The latter finding by Balota et al. is important, because it suggests that $\tau$ may be sensitive to top-down processes associated with actively using primes to help process degraded targets.

Indeed, changes in ex-Gaussian parameters have been taken to imply that a manipulation has influenced a certain cognitive process. That is, because different processes engage at times during an ongoing task, different processes should affect different portions of an RT distribution (e.g., Andrews \& Heathcote, 2001; Balota \& Spieler, 1999; Balota \& Yap, 2011; Heathcote et al. 2002; Hockley, 1984; Hohle, 1965; Rouder, 2005; Rouder \& Speckman, 2004; Wenger \& Gibson, 2004; Yap \& Seow, 2014). Some have suggested that the contributions of $\mu$ to $M_{\mathrm{RT}}$ may reflect selection and stimulus-driven processes, whereas the contributions of $\tau$ to $M_{\mathrm{RT}}$ may reflect slower top-down processes, such as retrieval; and that changes in the $\sigma$ component may reflect changes in difficulty of responding (e.g., Hohle, 1965; McGill, 1963; McGill \& Gibbon, 1965). Thus, employing an ex-Gaussian analysis can provide an alternate method to address how intertrial priming influences cognitive processes - that is, by examining how manipulations that influence PoP effects on $M_{\mathrm{RT}}$ also influence the shift $(\mu)$, skew $(\tau)$, and dispersion $(\sigma)$ of RTs. However, because there is debate over what specific processes could be mapped onto the ex-Gaussian parameters (Luce, 1986; Matzke \& Wagenmakers, 2009), we hold off on discussing this point until the General Discussion.

Recently, Kristjánsson and Jóhannesson (2014) fit the exGaussian to RTs collected in a feature (pop-out) search task and a conjunction search task, to examine how repetition priming of target features influenced the shift and skew of RTs. In the feature search task, the singleton target's color and/or the distractor color repeated, or neither color repeated; hence, there was no "switch" of the target and distractor colors across trials. They found that repetition priming of the target and distractor colors sped responding at the level of $M_{\mathrm{RT}}$ and influenced the $\mu$ and $\tau$ components, but not the $\sigma$ component. In the conjunction search task, the target was a white or black circle or square among a heterogeneous set of distractors, and this target repeated or did not repeat across trials. They found that repeating the target sped responding in $M_{\mathrm{RT}}$ and that this repetition priming influenced all three ex-Gaussian components. In short, Kristjánsson and Jóhannesson demonstrated that an ex-Gaussian analysis can reveal how intertrial priming influences different portions of an RT distribution.

In the present study, we used an ex-Gaussian analysis to examine how intertrial priming of pop-out search affected the 

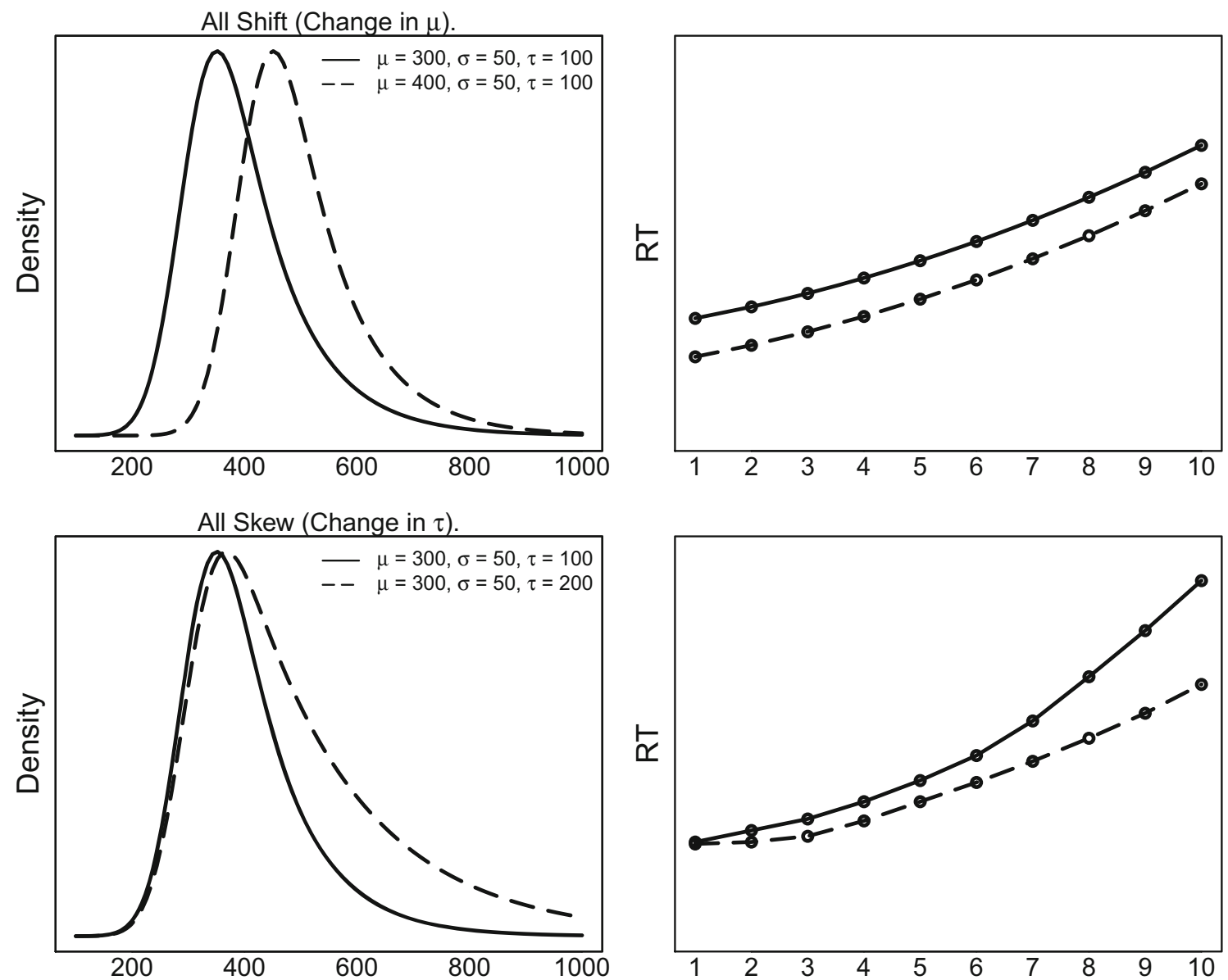

Shift and Skew (Change in $\mu$ and $\tau$ ).
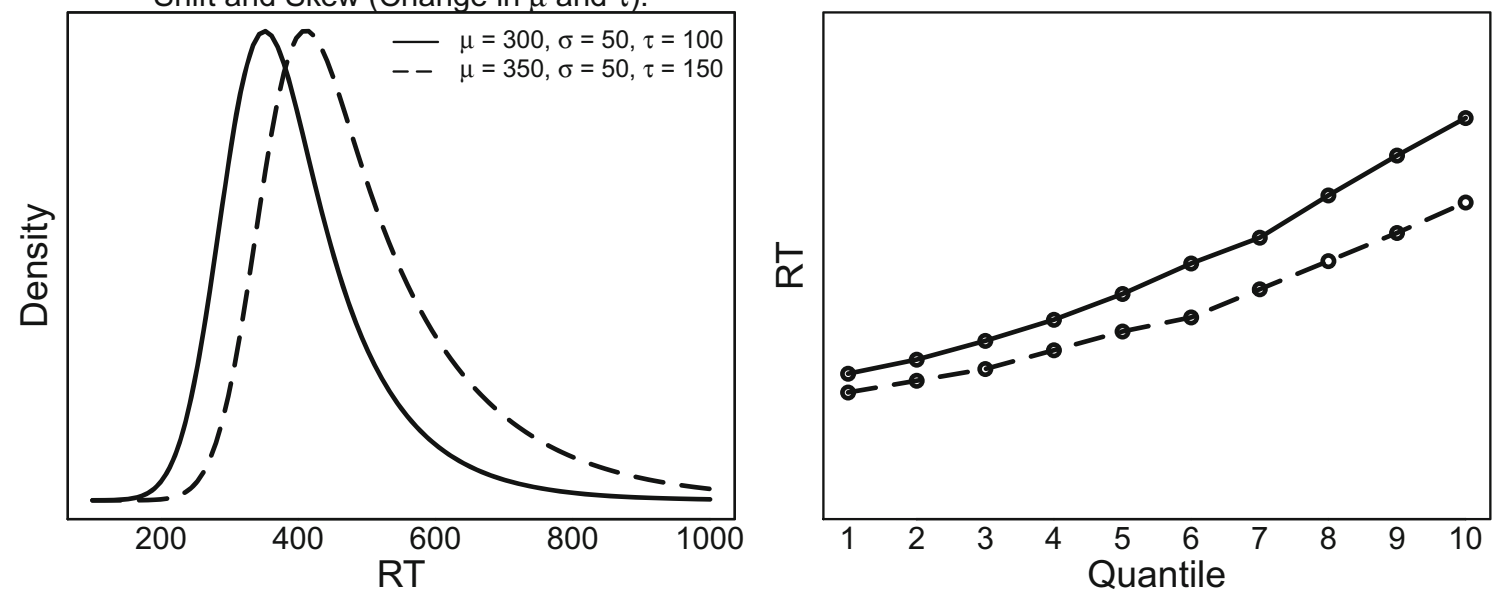

Fig. 1 Example ex-Gaussian distributions for two conditions(left), and the corresponding quantile plots(right) that can result if an experimental manipulation elicits a change in distribution shift(top), distribution skew(middle), or both shift and skew(bottom)

shift, skew, and dispersion of RT distributions. One difference between this study and Kristjánsson and Jóhannesson (2014) was that we used a typical PoP task in which the target color and distractor color repeated or switched across trials (the switch condition was not used in Kristjánsson \& Jóhannesson, 2014). A second difference is that this study examined how manipulations that influence the size of PoP effects on $M_{\mathrm{RT}}$ modulate the contributions of $\mu$ and $\tau$ to the PoP effects on $M_{\mathrm{RT}}$. Lastly, in addition to fitting the ex-Gaussian to the RTs, we also generated quantile plots to illustrate the influence of intertrial priming on the shift and skew of RTs (Fig. 1, right column). Quantile plots are created by rank-ordering a 
subject's RTs from fastest to slowest in each experimental condition. The RTs are separated into quantiles representing a percentage of the data and are plotted. Manipulations that produce differences in $M_{\mathrm{RT}}$ due to shifting (changes in $\mu$ ) result in little to no change between the conditions across quantiles, whereas manipulations that produce differences in $M_{\mathrm{RT}}$ due to skewing (changes in $\tau$ ) lead to increased differences between conditions across quantiles (see Fig. 3 in Balota \& Yap, 2011, as well as Tse, Hutchison, \& Li, 2011).

\section{Experiment 1}

In Experiment 1, we used a PoP task in which subjects searched a display for a variable-color singleton diamond among two homogeneously colored distractors and identified whether the target's left or right corner was missing. Across trials, the target color and distractor color repeated or switched, and it was expected that responding would be faster when the colors repeated. The question addressed was whether the PoP effect on $M_{\mathrm{RT}}$ would be due to distribution shifting $(\mu)$, skew $(\tau)$, or both, and how intertrial priming would influence the dispersion of RTs $(\sigma)$.

\section{Method}

Subjects Twenty University of Scranton undergraduates participated (16 female, four male; two left-handed). The subjects ranged in age from 18 to 20 years old $(M=$ $18.5, S D=0.69)$ and reported normal or corrected-to-normal vision.

Apparatus An Ishihara colorblindness test was used to assess color vision. The experiment was programmed using E-Prime version 2.0.10242 (Psychology Software Tools, Pittsburgh, PA) software on a Dell 755 computer with a Pentium Core 2 Duo processor with $1.96 \mathrm{~GB}$ RAM running at $2.33 \mathrm{GHz}$. Subjects sat approximately $60 \mathrm{~cm}$ from a Dell E178Fpv monitor with a resolution of $1,024 \times 768$ running at $60 \mathrm{~Hz}$, and a five-button response box was used to collect responses. A Cambridge Research Systems OptiCAL RS-232 photometer was used to take luminance readings from a small $\left(1^{\circ} \times 1^{\circ}\right)$ patch of each color on a black background.

Stimuli The search displays contained three diamonds $\left(1.1^{\circ} \times\right.$ $\left.1.1^{\circ}\right)$ presented on a uniform black background $\left(0.165 \mathrm{~cd} / \mathrm{m}^{2}\right.$, RGB: 0, 0, 0). Each diamond was missing its left or right corner (missing area subtended $0.14^{\circ}$ ). One diamond was the target and the other two were distractors, and the colors of the target and distractors were red $\left(20.44 \mathrm{~cd} / \mathrm{m}^{2}\right.$, RGB: 255 , $0,0)$ or green $\left(20.62 \mathrm{~cd} / \mathrm{m}^{2}\right.$, RGB: 10, 177, 31). A white fixation cross $\left(25.77 \mathrm{~cd} / \mathrm{m}^{2}, \mathrm{RGB}: 255,255,255\right)$ was presented throughout each trial to maintain ocular fixation. Each diamond appeared in one of 12 different locations on the circumference of an imaginary ellipse $\left(10^{\circ}\right.$ wide $\times 8^{\circ}$ high $)$ centered on the screen.

Procedures Subjects were tested individually in a closed soundproof room with normal illumination. Informed consent was obtained, and the Ishihara colorblindness was administered before instructions were given about the visual search task. Subjects were informed that they would see displays containing three diamonds and their task was to locate the color singleton and identify whether its left or right corner was missing as quickly and accurately as possible. Subjects pressed a left-hand key if the left corner was missing, and a right-hand key if the right corner was missing. Subjects were informed that the colors and the missing corner on the target were randomly chosen, so the next target's color and response were unpredictable.

Each trial began with a fixation display containing a white cross for $500 \mathrm{~ms}$. Then the target display was presented for 2,000 ms or until the subject made a response. The next trial began after a 100 -ms delay, but if the subject responded incorrectly or took longer than $2,000 \mathrm{~ms}$ to respond, a $500-\mathrm{Hz}$ tone was played during that $100-\mathrm{ms}$ delay. Subjects completed a practice block of 32 trials, followed by ten blocks of 96 trials each, and received a break after every block.

\section{Results}

Because distribution analyses require many trials for accurate parameter estimation, we used a criterion that a subject's overall accuracy had to be at least $80 \%$ correct to be included in the analyses. This resulted in the removal of five subjects. For the RT analyses, only trials on which a correct response was made on the current and preceding trials were used, which resulted in $10.8 \%$ of trials being removed for the remaining $n=15$ subjects. For the error analyses, only trials on which a correct response had been made on the preceding trial were used. Performance on each trial was sorted on the basis of the target and distractor colors on trials $n-1$ and $n$, to create a repeat condition (target and distractor colors repeated) and a reverse condition (target and distractor color switched). See Table 1 for the mean RTs, proportions of errors, and ex-Gaussian parameters. All mean RTs discussed here and presented in Table 1 are reported to the nearest millisecond, with the upper and lower limits of the $95 \%$ confidence intervals included in brackets. ${ }^{1}$

\footnotetext{
${ }^{1}$ The 95\% confidence intervals in the text and in Table 1 are based on the within-subjects error term (Eq.2, Hollands \& Jarmasz, 2010).
} 
Table 1 Mean response times, proportions of errors [ $p$ (error)], and mean ex-Gaussian parameters for the repeat and reverse conditions (trial $n-1$ to trial $n)$ in Experiments 1-3

\begin{tabular}{|c|c|c|c|c|c|c|c|}
\hline Exp & Intertrial Transition & Additional Factor Level & $M_{\mathrm{RT}}[95 \% \mathrm{CI}]$ & $p$ (Error) & $\mu[95 \% \mathrm{CI}]$ & $\tau[95 \% \mathrm{CI}]$ & $\sigma[95 \% \mathrm{CI}]$ \\
\hline \multirow[t]{3}{*}{ E1 } & Reverse & N/A & $814[802,826]$ & .064 & $608[596,620]$ & $205[194,216]$ & $79[72,85]$ \\
\hline & Repeat & & $722[710,734]$ & .046 & $541[529,553]$ & $181[170,192]$ & $55[48,61]$ \\
\hline & PoP & & $91[79,103]$ & .019 & $67[55,79]$ & $25[14,36]$ & $24[18,30]$ \\
\hline \multirow[t]{6}{*}{ E2 } & Reverse & Set Size 4 & $821[810,832]$ & .054 & $615[607,622]$ & $206[194,218]$ & $73[68,78]$ \\
\hline & Repeat & & $701[690,712]$ & .032 & $547[540,544]$ & $154[142,166]$ & $55[50,60]$ \\
\hline & PoP & & $120[109,131]$ & .022 & $68[61,75]$ & $52[40,64]$ & $17[12,22]$ \\
\hline & Reverse & Set Size 12 & $676[662,689]$ & .047 & $554[547,561]$ & $122[110,134]$ & $60[55,65]$ \\
\hline & Repeat & & $643[630,656]$ & .043 & $537[529,544]$ & $108[96,120]$ & $56[51,61]$ \\
\hline & PoP & & $32[18,45]$ & .003 & $17[10,24]$ & $14[2,25]$ & $4[-1,9]$ \\
\hline \multirow[t]{9}{*}{ E3 } & Reverse & RSI $300 \mathrm{~ms}$ & $942[931,953]$ & .075 & $739[720,760]$ & $203[187,218]$ & $114[100,127]$ \\
\hline & Repeat & & $803[792,814]$ & .040 & $612[591,633]$ & $191[175,206]$ & $63[50,76]$ \\
\hline & PoP & & $139[128,113]$ & .036 & $126[105,147]$ & $12[-3,28]$ & $51[38,64]$ \\
\hline & Reverse & RSI $600 \mathrm{~ms}$ & $842[831,853]$ & .054 & $642[621,663]$ & $200[184,216]$ & $82[68,95]$ \\
\hline & Repeat & & $739[728,750]$ & .029 & $569[548,591]$ & $170[154,186]$ & $55[42,68]$ \\
\hline & PoP & & $102[91,113]$ & .025 & $73[52,94]$ & $29[13,44]$ & $27[14,40]$ \\
\hline & Reverse & RSI $2,000 \mathrm{~ms}$ & $789[778,800]$ & .025 & $622[601,643]$ & $167[151,182]$ & $76[62,89]$ \\
\hline & Repeat & & $732[721,743]$ & .015 & $577[556,598]$ & $155[139,170]$ & $63[49,76]$ \\
\hline & PoP & & $57[46,68]$ & .009 & $45[24,66]$ & $12[-4,28]$ & $13[0,26]$ \\
\hline
\end{tabular}

The $95 \%$ confidence intervals are based on the within-subjects error term (Eq. 2, Hollands \& Jarmasz, 2010).

Response times Responding was faster in the repeat condition than in the reverse condition $[t(14)=11.88, S E=7.69, p<$ $\left..001, d=0.79^{2}\right]$, revealing a PoP effect of $91 \mathrm{~ms}[79,103]$.

RT distribution Each subject's data in the repeat and reverse conditions were fit to an ex-Gaussian function using RTSYS (Heathcote, 1996), which uses continuous maximum likelihood estimation to obtain ex-Gaussian parameters. (For the mean numbers of trials per subject in all conditions of each experiment, see Table 2.) All fits converged within 500 iterations. The parameter estimates for $\mu, \sigma$, and $\tau$ were compared between the repeat and reverse conditions. For $\mu$, the reverse condition was greater than the repeat condition $[t(14)=08.72$, $S E=7.68, p<.001, d=0.94]$, revealing a PoP effect on $\mu$ of $67 \mathrm{~ms}[55,19]$. For $\tau$, the reverse condition was also greater than the repeat condition $[t(14)=3.48, S E=7.06, p=.004, d=$ $0.43]$, revealing a PoP effect on $\tau$ of $25 \mathrm{~ms}[14,36]$. Lastly, for $\sigma$, the reverse condition was greater than the repeat condition $[t(14)=5.78, S E=4.25, p<.001, d=1.18]$, revealing a PoP effect on $\sigma$ of $24 \mathrm{~ms}[18,30]$. Note that the PoP effect on $M_{\mathrm{RT}}$ (91 $\mathrm{ms})$ is the sum of the PoP effects on $\mu(67 \mathrm{~ms})$ and $\tau$

\footnotetext{
${ }^{2}$ Cohen's $d$ for paired $t$ tests was calculated using the average standard deviation $\left(s_{\text {avg }}\right)$; see Eq.11.9 in Cumming (2012): $s_{\text {avg }}=\sqrt{\frac{s_{A}^{2}+s_{B}^{2}}{2}}$. The variances in the numerator come from the two conditions compared in the $t$ test.
}

(25 ms), suggesting that intertrial priming influenced both distribution shifting and skew.

To illustrate these effects graphically, in the repeat and reverse conditions each subject's data were sorted from fastest to slowest. The first $10 \%$ of each subject's data were averaged, followed by the second $10 \%$, the third $10 \%$, and so forth, resulting in ten quantiles. The quantiles were then averaged across subjects and plotted in Fig. 2. As can be seen in this figure, the PoP effects were smallest at the earliest quantiles and increased across quantiles, suggesting that intertrial priming influenced both the distribution shifting and skew, consistent with the ex-Gaussian analysis.

Errors Fewer errors were made in the repeat condition $(M=.046)$ than in the reverse condition $(M=.064)[t(14)=$ 3.90, $S E=.0048, p=.002, d=0.58]$.

\section{Discussion}

Experiment 1 demonstrated that both distribution shifting $(\mu)$ and skewing $(\tau)$ contributed to the PoP effect on $M_{\mathrm{RT}}$, consistent with Kristjánsson and Jóhannesson (2014). We also found that repeating versus switching the target and distractor colors across trials influenced the $\sigma$ component, with larger values of $\sigma$ in the reverse than in the repeat condition. It is worth noting that this result differs from that of Kristjánsson and Jóhannesson, who did not find an influence of priming on $\sigma$, 
Table 2 Mean numbers of trials per subject in the repeat and reverse conditions

\begin{tabular}{|c|c|c|c|c|c|c|}
\hline \multirow{2}{*}{$\begin{array}{l}\text { Intertrial } \\
\text { Transition }\end{array}$} & \multirow[b]{2}{*}{ Experiment 1} & \multicolumn{2}{|l|}{ Experiment 2} & \multicolumn{3}{|l|}{ Experiment 3} \\
\hline & & Set Size 4 & Set Size 12 & RSI 300 & RSI 600 & RSI 2,000 \\
\hline Repeat & $418.6(26.7)$ & $219.9(16.6)$ & $213.4(16.6)$ & $165.9(13.1)$ & $179.3(13.0)$ & $173.4(18.3)$ \\
\hline Reverse & $429.0(34.8)$ & $212.9(17.2)$ & $219.6(16.0)$ & $175.4(16.0)$ & $170.0(25.7)$ & $182.2(9.3)$ \\
\hline
\end{tabular}

Values in parentheses are the standard deviations.

but they did not include a feature-switch condition, which may be critical for this result to arise.

Having established that distribution shifting $(\mu)$ and skewing $(\tau)$ contributed to the PoP effects on $M_{\mathrm{RT}}$, in the following two experiments we examined how manipulations that should influence the PoP effects would also moderate the contributions of $\mu$ and $\tau$ to the PoP effect on $M_{\mathrm{RT}}$.

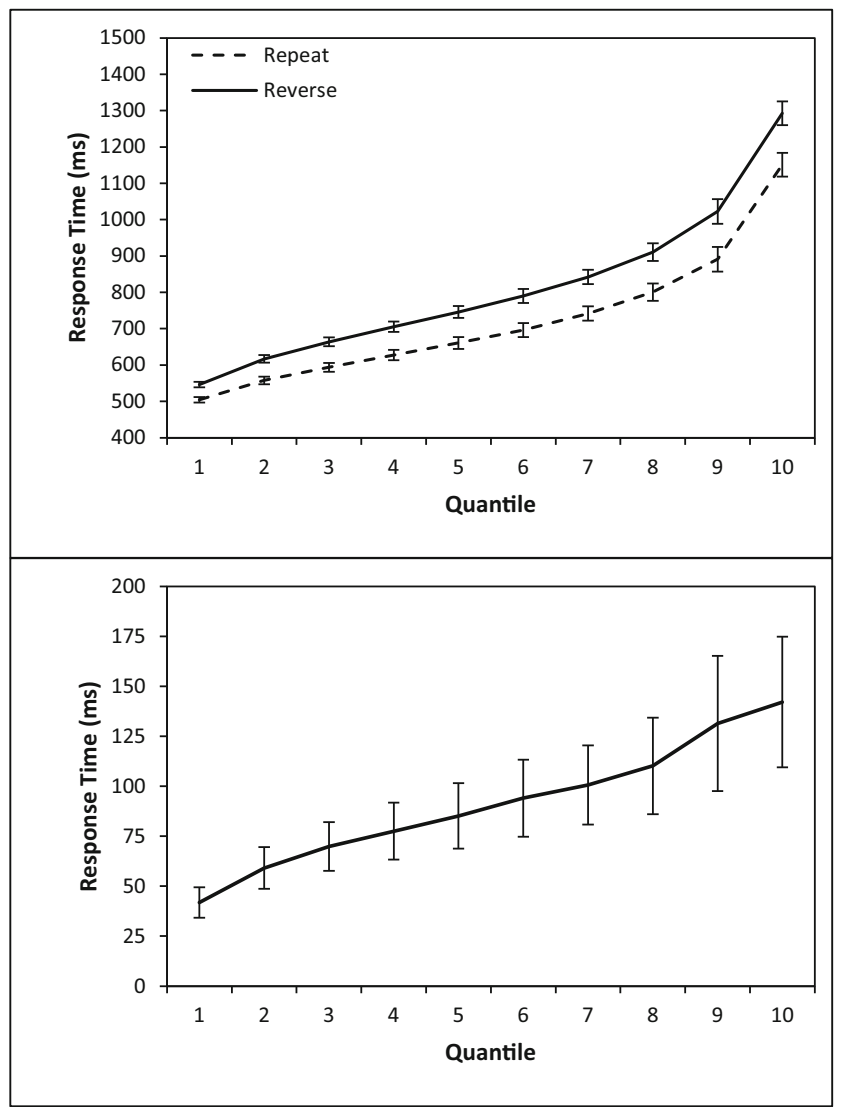

Fig. 2 Quantile plots for the repeat and reverse conditions(top) and priming of pop-out (PoP) effects(bottom) in Experiment 1. Error bars are $95 \%$ confidence intervals based on the standard errors of the means for paired differences between the repeat and reverse conditions at each quantile: $S E M_{k l}^{\text {pairedDiff }}=\sqrt{\frac{1}{n(n-1)} \sum_{i=1}^{n}\left(d_{i}-\bar{d} .\right)^{2}}$, where $d_{\mathrm{i}}$ is the difference between the reverse and repeat conditions for subject $i$, and $\bar{d}$. is the mean difference across subjects (Franz \& Loftus, 2012)

\section{Experiment 2}

In Experiment 2, the search display set size was manipulated, to examine how varying the number of nontarget items in the display moderated the contributions of $\mu$ and $\tau$ to the PoP effect on $M_{\mathrm{RT}}$. Experiment 2 was similar to an experiment conducted by Meeter and Olivers (2006), who observed smaller PoP effects in displays with larger set sizes. They attributed this decreased PoP effect to less ambiguity about what item was the target, that is, with more homogeneously colored distractors in the display, it was easier to determine which item was the target. Given that a singleton target is more salient with a larger set size, target selection should be easier. Hence, if changes in ex-Gaussian parameters imply changes in cognitive processes, we should find that set size also moderates the relative contributions of $\mu$ and $\tau$ to the PoP effects on $M_{\mathrm{RT}}$, and should also influence the size of the PoP effect on $\sigma$.

\section{Method}

Unless noted, all methods were the same as in Experiment 1.

Subjects Twenty undergraduates participated (18 female, two male; two left-handed). Subjects ranged in age from 18 to 20 years old $(M=18.25, S D=0.55)$ and reported normal or corrected-to-normal color vision.

Stimuli The stimuli were identical to those in Experiment 1, with one exception. In Experiment 2, the search display size (set size) was either four or 12 diamonds.

Design The experiment was based on a 2 (Set Size: 4 vs. 12 diamonds) $\times 2$ (repeat vs. reverse) within-subjects factorial design. Set size was blocked and counterbalanced for order across subjects; subjects completed 32 practice trials and then five blocks of 96 trials for each set size.

\section{Results}

The data were handled in the same way as in Experiment 1, which resulted in the removal of one subject's data due to 
accuracy being less than $80 \%$. Of the remaining $n=19$ subjects, $8.5 \%$ of the data were removed due to error.

Response times A 2 (Set Size: 4 vs. 12 diamonds) $\times 2$ (repeat vs. reverse) ANOVA on $M_{\mathrm{RT}} \mathrm{s}$ yielded a main effect of set size $\left[F(1,18)=42.87, M S E=4,596.78, p<.001, f=1.54^{3}\right]$, due to faster responding to 12 -item displays $(M=660 \mathrm{~ms}[637,683])$ than to four-item displays $(M=761 \mathrm{~ms}[738,784])$. The overall 76-ms $[66,85]$ PoP effect was significant $[F(1,18)=$ $141.52, M S E=780.71, p<.001, f=2.80]$ and was modulated by set size $[F(1,18)=67.33, M S E=540.95, p<.001, f=$ 1.93]. The interaction indicated that the $120-\mathrm{ms}[109,131]$ PoP effect for the four-item displays $[t(18)=12.12, S E=$ $9.90, p<.001, d=1.47]$ was larger than the 32-ms $[18,45]$ PoP effect for the 12-item displays $[t(18)=5.08, S E=6.40$, $p<.001, d=0.32]$, which replicated the results of Meeter and Olivers (2006).

RT distribution Separate 2 (Set Size) $\times 2$ (Repeat vs. Reverse) ANOVAs were carried out on each ex-Gaussian parameter. For $\mu$, there was a main effect of set size $[F(1,18)=$ 23.20, $M S E=1,071.87, p<.001, f=1.14]$, due to a smaller estimate of $\mu$ in 12-item displays ( $M=545 \mathrm{~ms}[534,556])$ than in four-item displays $(M=581 \mathrm{~ms}[569,592])$. The 43-ms [36, 50] PoP effect was significant $[F(1,18)=85.96, M S E=$ 400.22, $p<.001, f=2.19$ ] and was modulated by set size $[F(1,18)=55.72, M S E=218.80, p<.001, f=1.76]$. The interaction suggests that the 68-ms $[61,75]$ PoP effect for the four-item displays $[t(18)=10.59, S E=6.41, p<.001, d=$ 1.29] was larger than the 17-ms [10,24] PoP effect for the 12item displays $[t(18)=3.51, S E=4.90, p=.002, d=0.27]$.

For $\tau$, there was a main effect of set size $[F(1,18)=30.78$, $M S E=2,614.82, p<.001, f=1.31]$, due to a larger estimate of $\tau$ in 12-item displays $(M=180 \mathrm{~ms}[162,197])$ than in fouritem displays ( $M=115 \mathrm{~ms}[97,132])$. The 33-ms [23,42] PoP effect was significant $[F(1,18)=29.56, M S E=712.54, p<$ $.001, f=1.28]$ and was modulated by set size $[F(1,18)=$ 11.14, MSE $=607.47, p=.004, f=0.79]$. The 52-ms [40, 64] PoP effect for four-item displays $[t(18)=5.56, S E=$ 9.37, $p<.001, d=1.08]$ was larger than the 14-ms $[2,26]$ effect for 12-item displays $[t(18)=2.02, S E=7.15, p=$ $.059, d=0.28$ ].

For $\sigma$, the main effect of set size was not significant $[F(1$, $18)=2.35, M S E=273.89, p=.143, f=0.36]$, though the estimate of $\sigma$ was smaller for 12-item displays ( $M=58 \mathrm{~ms}[52$, $64])$ than for four-item displays $(M=64 \mathrm{~ms}[58,70])$. The 11$\mathrm{ms}[7,15]$ PoP effect was significant $[F(1,18)=17.89, M S E=$ $126.48, p=.001, f=1.00]$ and was modulated by set size $[F(1,18)=6.89, M S E=121.27, p=.017, f=0.62]$. The $17-\mathrm{ms}$

\footnotetext{
${ }^{3}$ Cohen's $f$ was calculated using Eq. 8.2.22 in Cohen (1988): $f=\sqrt{\frac{\eta^{2}}{1-\eta^{2}}}$
}

$[12,22]$ PoP effect for four-item displays $[t(18)=4.81, S E=$ $3.65, p<.001, d=0.88]$ was larger than the 4-ms $[-1,9] \mathrm{PoP}$ effect for 12-item displays $[t(18)=1.20, S E=3.57, p=.247$, $d=0.26]$.

Quantile plots for the repeat and reverse conditions and the PoP effects for each set size are displayed in Fig. 3. As can be seen in that figure, PoP effects were smallest at the shortest quantiles and increased in magnitude across quantiles, suggesting influences of distribution shifting and skew on the PoP effect on $M_{\mathrm{RT}}$, consistent with the ex-Gaussian analysis. Importantly, the increase in the PoP effects across quantiles was less pronounced for the set size of 12 items, consistent with the smaller PoP effect on $\tau$ for the 12-item displays.

Errors A 2 (Set Size) $\times 2$ (Repeat vs. Reverse) ANOVA yielded a PoP effect $[M=.012[.004, .021], F(1,18)=10.41$, $M S E=.001, p=.005, f=0.76]$ that was moderated by set size $[F(1,18)=4.81, M S E=.001, p=.042, f=0.52]$. The PoP effect was larger for four-item displays $[t(18)=3.48, S E=$

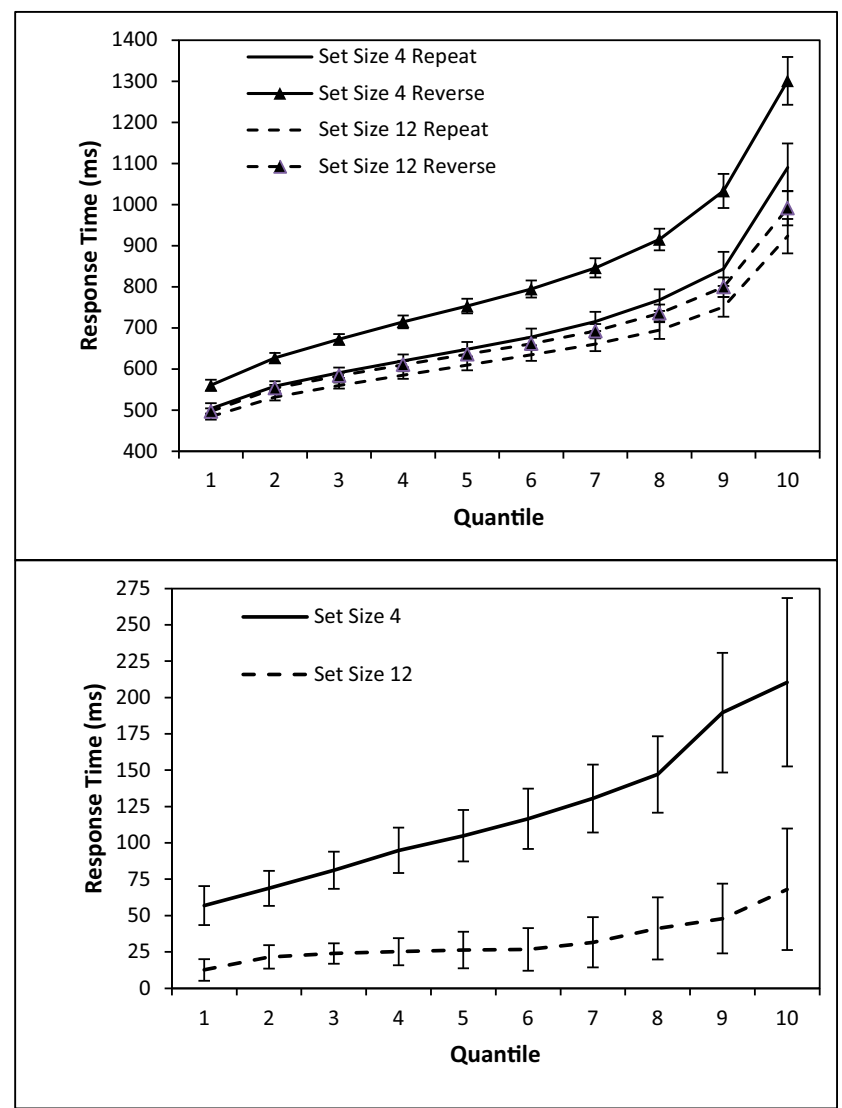

Fig. 3 Quantile plots for the repeat and reverse conditions(top) and PoP effects(bottom) for each set size in Experiment 2. Error bars are 95\% confidence intervals based on the standard errors of the means for paired differences between the repeat and reverse conditions at each quantile: $S E M_{k l}^{\text {pairedDiff }}=\sqrt{\frac{1}{n(n-1)} \sum_{i=1}^{n}\left(d_{i}-\bar{d}\right)^{2}}$, where $d_{\mathrm{i}}$ is the difference between the reverse and repeat conditions for subject $i$, and $\bar{d}$. is the mean difference across subjects (Franz \& Loftus, 2012) 
$.006, p=.003, d=0.68]$ than for 12 -item displays, $t<1$. The set size effect was not significant, $F<1$.

\section{Discussion}

The results of Experiment 2 replicated those of Experiment 1 by showing that distribution shifting $(\mu)$ and skew $(\tau)$ contributed to PoP effects on $M_{\mathrm{RT}}$, and also replicated the results of Meeter and Olivers (2006) by showing that set size moderated the PoP effect on $M_{\mathrm{RT}}$. Importantly, the larger set size was associated with smaller contributions of $\mu$ and $\tau$ to the PoP effect on $M_{\mathrm{RT}}$ and with smaller PoP effect on $\sigma$. Larger set sizes reduce the ambiguity over which item is the target and should reduce or eliminate the need for postselection verification that a selected item is the target, but would affect the need to select a target. As such, because the manipulation of set size modulated the PoP effects on all three ex-Gaussian parameters, this might suggests that several processes underlie intertrial priming, consistent with Lamy et al. (2010) dual-stage account. However, we will hold off on further discussion of this point until the General Discussion.

\section{Experiment 3}

In Experiment 3, the response-stimulus interval (RSI) between the response on trial $n-1$ and presentation of the display on trial $n$ was manipulated. Subjects were presented with the same displays as in Experiment 1, but the time between their response and the appearance of the next display was the same as in Experiment $1(600 \mathrm{~ms})$, was shorter $(300 \mathrm{~ms})$, or was longer $(2,000 \mathrm{~ms})$. This was intended to influence the size of the PoP effects on $M_{\mathrm{RT}}$, as well as the size of the PoP effects on the ex-Gaussian parameters. Priming effects on $M_{\mathrm{RT}}$ tend to decrease as the prime-to-target onset increases. We expected that as RSI increased, activation of the trial $n-1$ target color would decrease and would have less influence on selection for trial $n$. Thus, an increase in RSI should be accompanied by a decrease in the PoP effects on $M_{\mathrm{RT}}$, as well as a change in the PoP effects on $\mu$ and $\sigma$, but with little change to $\tau$.

\section{Method}

Unless noted, all methods were the same as in Experiment 1.

Subjects Twenty-four University of Scranton undergraduates participated (20 female, four male; four left-handed). Subjects ranged in age from 18 to 21 years old $(M=18.50, S D=0.83)$ and reported normal or corrected-to-normal vision.

Design The experiment was based on a 3 (RSI: 300, 600, 2, $000 \mathrm{~ms}) \times 2$ (repeat vs. reverse) within-subjects factorial design. RSI was blocked and counterbalanced for order across subjects, who completed 32 practice trials at the 600 -ms RSI and then five blocks of 96 trials each for each RSI.

\section{Results}

The data were handled in the same way as in Experiments 1 and 2, which resulted in the removal of three subjects' data due to accuracy below $80 \%$. Of the remaining $n=21$ subjects, $7.9 \%$ of the data were removed due to error.

Response times A 3 (RSI) $\times 2$ (repeat vs. reverse) ANOVA revealed a main effect of RSI $[F(2,40)=14.13, M S E=10$, $068.18, p<.001, f=0.84]$. Responding was significantly slower at the $300-\mathrm{ms}$ interval $(M=873 \mathrm{~ms}[841,905])$ than at the $600-\mathrm{ms} \operatorname{RSI}(M=791 \mathrm{~ms}[759,823], p=.002)$ and the 2 , 000-ms RSI $(M=760 \mathrm{~ms}[728,792], p<.001)$; the difference in $M_{\mathrm{RT}}$ between the 600-ms and 2,000-ms RSIs was not significant $(p=.123)$. The $99-\mathrm{ms}[81,117] \mathrm{PoP}$ effect was significant $[F(1,20)=67.72, M S E=4,591.10, p<.001, f=1.84]$ and was moderated by RSI $[F(2,40)=28.75, M S E=612.73$, $p<.001, f=1.20]$. The interaction shows that the PoP effect was largest at the 300-ms RSI $[t(20)=7.63, S E=18.18, p<$ $.001, d=1.18]$ and decreased at the 600-ms RSI $[t(20)=8.16$, $S E=12.54, p<.001, d=1.23]$ and the $2,000-\mathrm{ms}$ RSI $[t(20)=$ $7.00, S E=8.14, p<.001, d=0.58]$.

RT distributions Separate 3 (RSI) $\times 2$ (repeat vs. reverse) ANOVAs were carried out on each ex-Gaussian parameter. For $\mu$, the main effect of RSI was significant $[F(2,40)=$ $10.03, M S E=7,532.89, p<.001, f=0.71]$. Estimates of $\mu$ were significantly greater at the $300-\mathrm{ms}$ RSI $(M=675 \mathrm{~ms}$ $[647,703])$ than at the $600-\mathrm{ms}$ RSI $(M=605 \mathrm{~ms}[577,632]$, $p=.001)$ and the 2,000-ms RSI $(M=599 \mathrm{~ms}$ [571,627], $p=$ .001 ); the difference between the 600- and 2,000-ms RSIs was not significant $(p=.693)$. The $81-\mathrm{ms}[66,96]$ PoP effect was significant $[F(1,20)=61.30, M S E=3,398.86, p<.001, f=$ $1.75]$ and was moderated by RSI $[F(2,40)=8.41, M S E=$ $2145.99, p=.001, f=0.65]$. The interaction indicates that the PoP effect was largest at the 300-ms RSI $[t(20)=5.13$, $S E=24.62, p<.001, d=1.12]$ and decreased at the $600-\mathrm{ms}$ RSI $[t(20)=7.83, S E=9.32, p<.001, d=1.10]$ and the 2,000ms RSI $[t(20)=7.10, S E=6.30, p<.001, d=0.54]$.

For $\tau$, the main effect of RSI was significant $[F(2,40)=$ $6.61, M S E=2,130.04, p=.003, f=0.57]$. Estimates of $\tau$ were significantly lower at the 2,000-ms RSI $(M=161 \mathrm{~ms}[146$, 176]) than at the 300-ms RSI $(M=197 \mathrm{~ms}[182,212], p=$ $.003)$ and the $600-\mathrm{ms}$ RSI $(M=185 \mathrm{~ms}[170,200], p=.004)$; the difference between the $300-\mathrm{ms}$ and $600-\mathrm{ms}$ RSIs was not significant $(p=.312)$. The 18-ms [11,25] PoP effect was significant $[F(1,20)=13.25, M S E=756.40, p=.002, f=0.81]$, but the interaction was not $[F<1]$. However, the PoP effect was not significant at the 300 -ms RSI $[t<1]$ or the $2,000-\mathrm{ms}$ RSI $[t(20)=1.37, S E=8.62, p=.186, d=0.23]$, but was 
significant at the 600-ms RSI $[t(20)=3.95, S E=7.45, p=$ $.001, d=0.53]$.

For $\sigma$, the main effect of RSI was significant $[F(2,40)=$ $4.19, M S E=1,280.99, p=.022, f=0.46]$. The estimate of $\sigma$ was significantly greater at the 300 -ms RSI $(M=89 \mathrm{~ms}[77$, $100])$ than at the 600-ms RSI $(M=69 \mathrm{~ms}[57,80], p=.008)$, and marginally greater than at the $2,000-\mathrm{ms}$ RSI $(M=69 \mathrm{~ms}$ $[57,80], p=.078)$; the difference between the 600 - and 2,000ms RSIs was not significant $(p=.927)$. The 30 -ms $[20,40]$ PoP effect was significant $[F(1,20)=21.54, M S E=1,312.81$, $p<.001, f=1.04]$ and was moderated by RSI $[F(2,40)=4.42$, $M S E=877.44, p=.018, f=0.47]$. The interaction shows that the PoP was largest at the 300-ms RSI $[t(20)=3.34, S E=$ $15.16, p=.003, d=0.97]$ and decreased at the 600 -ms RSI $[t(20)=4.16, S E=6.43, p<.001, d=1.11]$ and the $2,000-\mathrm{ms}$ RSI $[t(20)=2.74, S E=4.58, p=.013, d=0.76]$.

Quantile plots for the repeat and reverse conditions at each RSI and for the PoP effects are displayed in Fig. 4. As can be seen in the figure, the PoP effects were smallest at the shortest quantiles and increased in magnitude across quantiles, consistent with the ex-Gaussian analysis. The increase in PoP effects across quantiles was more pronounced at the 600-ms RSI (consistent with Exp.1) and, surprisingly, at the 300-ms than at the 2,000-ms RSI.

Errors A $3(\mathrm{RSI}) \times 2$ (Repeat vs. Reverse) ANOVA revealed a main effect of RSI $[F(2,40)=15.75, M S E=.001, p<.001$, $f=0.89]$. Responses were less accurate at the 300 -ms RSI $(M=.057)$, and became more accurate at the $600-\mathrm{ms}$ RSI $(M=.042)$ and the 2,000 -ms RSI $(M=.020)$. The PoP effect was significant $[M=.023, F(1,20)=17.44, M S E=.001, p<$ $.001, f=0.87]$ and was moderated by $\operatorname{RSI}[F(2,40)=5.52$, $M S E=.001, p=.008, f=0.52]$. The interaction indicates that the effect was largest at the 300-ms RSI $[t(20)=4.18, S E=$ $.008, p<.001, d=0.71]$ and decreased at the 600 -ms RSI $[t(20)=2.90, S E=.008, p=.009, d=0.65]$ and the $2,000-\mathrm{ms}$ RSI $[t(20)=2.84, S E=.003, p=.010, d=0.52]$.

\section{Discussion}

Experiment 3 replicated Experiments 1 and 2 by showing that both distribution shift $(\mu)$ and skew $(\tau)$ contributed to the PoP

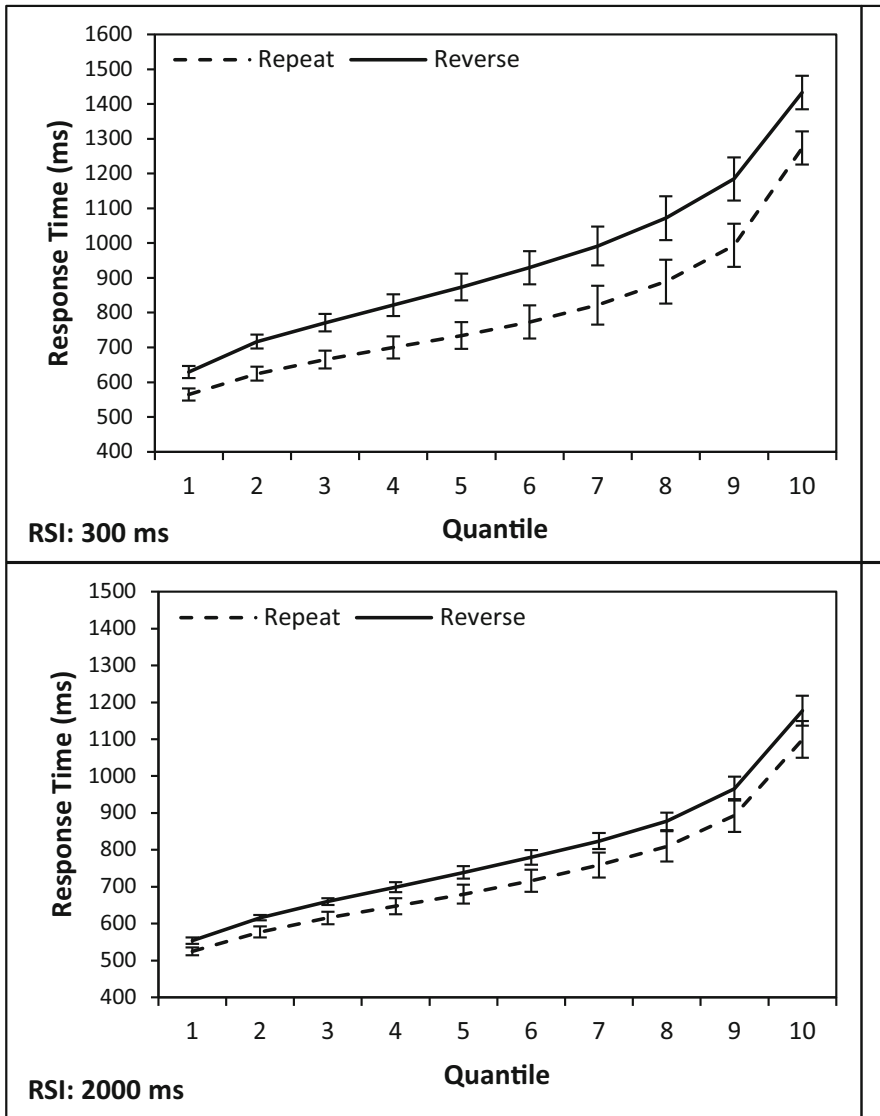

Fig. 4 Quantile plots for the repeat and reverse conditions for the 300ms (upper left), 600-ms (upper right), and 2,000-ms (lower left) RSIs, and the PoP effects for each RSI(lower right) in Experiment 3. Error bars are $95 \%$ confidence intervals based on the standard errors of the means for paired differences between the repeat and reverse conditions at each

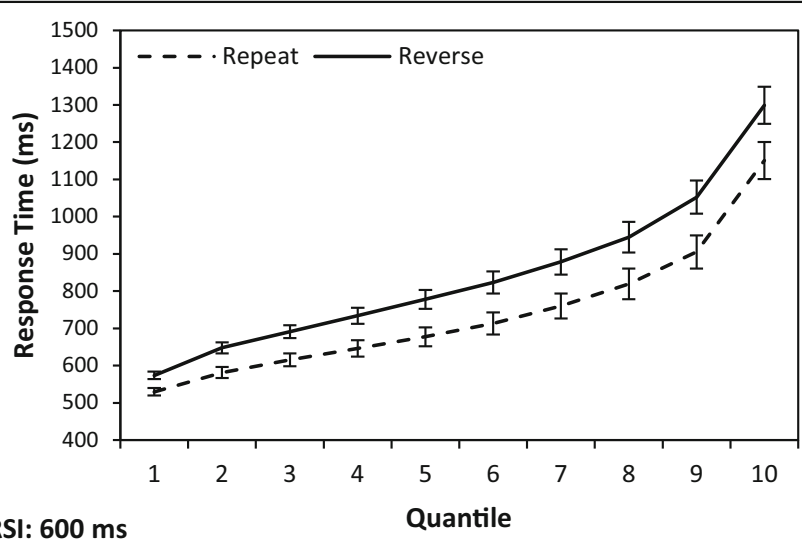

RSI: $600 \mathrm{~ms}$

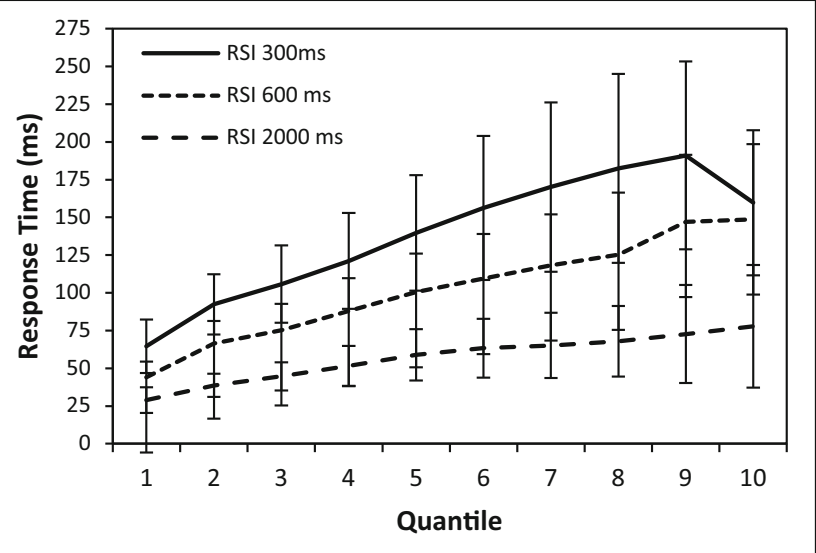

quantile: $S E M_{k l}^{\text {pairedDiff }}=\sqrt{\frac{1}{n(n-1)} \sum_{i=1}^{n}\left(d_{i}-\bar{d}\right)^{2}}$, where $d_{\mathrm{i}}$ is the difference between the reverse and repeat conditions for subject $i$, and $\bar{d}$. is the mean difference across subjects (Franz \& Loftus, 2012) 
effects on $M_{\mathrm{RT}}$. The results of Experiment 3 are important because they also revealed an influence of RSI on the intertrial priming effects. Specifically, and as predicted, the PoP effects on $M_{\mathrm{RT}}$ decreased as RSI increased, suggesting a reduced influence of trial $n-1$ as the RSI increased. Perhaps more importantly, the decrease in the $M_{\mathrm{RT}}$ PoP effects was accompanied by decreased PoP effects on $\mu$ and $\sigma$ as RSI increased; however, the PoP effects on $\tau$ were relatively unaffected as RSI increased. Taken together, the results of Experiment 3 indicate that the decrease in a target feature's influence over increasing delays has a stronger influence on the central tendency and dispersion of the RT distribution, but little influence on the skew.

\section{General discussion}

In the present study, we examined intertrial priming's influences on the shift, skew, and dispersion of RTs in a pop-out search task. Each experiment demonstrated how both distribution shift $(\mu)$ and skew $(\tau)$ contributed to the PoP effect on $M_{\mathrm{RT}}$, and also demonstrated a PoP effect on the dispersion $(\sigma)$ of RTs; hence, intertrial priming influenced all three exGaussian parameters. Importantly, in Experiments 2 and 3 we used manipulations intended to moderate the size of the PoP effects on $M_{\mathrm{RT}}$ in order to examine the relative changes to intertrial priming's influences on the shift, skew, and dispersion of the RT distributions. In Experiment 2, larger display set sizes were associated with a smaller PoP effect on $M_{\mathrm{RT}}$ that was driven by a decrease in the contributions of both $\mu$ and $\tau$. In Experiment 3, longer delays between the response on trial $n-1$ and the display on trial $n$ were associated with smaller PoP effects on $M_{\mathrm{RT}}$ that were driven primarily by a decreased contribution of $\mu$. The results of this study indicate that intertrial priming influences all three ex-Gaussian components of the underlying RT distributions in pop-out search tasks. As will be explained below, the results suggest that multiple cognitive processes underlie intertrial priming effects, but before discussing the results of the ex-Gaussian analyses, we will address how the results are inconsistent with the notion that one process is influenced by intertrial priming.

Initial explanations of intertrial priming proposed that implicitly encoded target features biased selection toward that same feature on trialn (Maljkovic \& Nakayama, 1994, 1996). The present study is inconsistent with such pureselection accounts, because in Experiment 3 we found PoP effects on $M_{\mathrm{RT}}$ after 2,000 ms. Given target activation decays over time, finding PoP effects after such a long delay suggests that postselection processes were operating. In contrast, postselection accounts propose intertrial priming influences the retrieval of previous instances of a target (Hillstrom, 2000; Huang et al. 2004; Huang \& Pashler, 2005; Thomson \& Milliken, 2011, 2012, 2013). The present study is also inconsistent with this account, because in Experiment 2 we observed PoP effects when the set size was 12 items, even though target ambiguity was reduced and there was little need to retrieve prior instances of targets to verify that a selected item was the target. Thus, the results are more consistent with Lamy et al. (2010) dual-stage model of intertrial priming and with Kristjánsson and Campana’s (2010) conclusion that intertrial priming influences at least two cognitive processes: selection and postselection retrieval.

How might the ex-Gaussian analyses of RTs provide evidence that more than one cognitive process was influenced by intertrial priming? An assumption in prior studies was that changes in the estimates of $\mu$ and $\tau$ reflect changes in separate cognitive processes. Specifically, changes in $\mu$ were thought to reflect changes in faster, stimulus-driven processes (e.g., selection, perception), whereas changes in $\tau$ were thought to reflect changes in slower, later-occurring, top-down processes (e.g., retrieval, response selection; e.g., Andrews \& Heathcote, 2001; Balota \& Spieler, 1999; Balota \& Yap, 2011; Balota et al. 2008; Heathcote et al. 2002; Hockley, 1984; Hohle, 1965; McGill, 1963; McGill \& Gibbon, 1965; Rouder, 2005; Rouder \& Speckman, 2004; Van Zandt, 2000; Yap \& Seow, 2014). These assumptions are based on which areas of the RT distribution are estimated by $\mu$ and $\tau .{ }^{4}$ Because $\mu$ estimates the location of the Gaussian portion, which is associated with the faster responses, and because stimulus-driven processes operate quickly, these processes may be reflected by changes in $\mu$. Similarly, because $\tau$ estimates the skewed (exponential) portion, which is associated with slower responding, and because top-down processes engage more slowly, these processes may be associated with changes in $\tau$. In short, changes in $\mu$ are thought to reflect stimulus-driven processes, and changes in $\tau$ are thought to reflect top-down processes.

If intertrial priming facilitates both early selection and episodic retrieval processes, an ex-Gaussian analysis should reveal contributions of both $\mu$ and $\tau$ to the PoP effects on $M_{\mathrm{RT}}$, which was observed in each experiment reported in this study. Stated differently, the PoP effects on $\mu$ and $\tau$ suggest that at least two cognitive processes were influenced by intertrial priming of target and distractor features. Importantly, the manipulations used in Experiments 2 and 3 should have influenced selection and/or postselection retrieval processes and should have elicited corresponding changes in the PoP effects on $\mu$ and $\tau$. In Experiment 2, when the set size was four items, and target ambiguity was about the same as in Experiment 1, both $\mu$ and $\tau$ contributed to the PoP effect on $M_{\mathrm{RT}}$. In contrast, for the set size of 12 items, target ambiguity was low, making the target not only easier to select, but making episodic retrieval and target

\footnotetext{
${ }^{4}$ We do not discuss what processes may be reflected in $\sigma$, because this parameter is rarely discussed in the literature.
} 
Table 3 Mean estimates of the EZ-diffusion model parameters in the repeat and reverse conditions and $t$ test results for Experiment 1

\begin{tabular}{llcrr}
\hline Intertrial Transition & Statistic & Drift Rate $(v)$ & Separation $(a)$ & Nondecision Time $\left(T_{\text {er }}\right)$ \\
\hline Reverse & $M_{\text {Reverse }}(S E)$ & $.211(.014)$ & $.140(.006)$ & $.514(.020)$ \\
Repeat & $M_{\text {Repeat }}(S E)$ & $.241(.013)$ & $.137(.005)$ & $.454(.017)$ \\
& $t(S E M)$ & $-5.665(.005)$ & $1.678(.002)$ & $8.892(.007)$ \\
& $p$ & $<.001$ & .116 & $<.001$ \\
& Cohen's $d$ & 0.568 & 0.173 & 0.838 \\
\hline
\end{tabular}

Each $t$ test $(d f=14)$ compared the mean estimate in the reverse condition to the mean estimate in the repeat condition.

verification less necessary. Thus, reduced target ambiguity should result in smaller PoP effects on $\mu$ (because selection was easier) and $\tau$ (because episodic retrieval was less necessary) for the set size of 12 items, which was observed. In Experiment 3, PoP effects on $M_{\mathrm{RT}}$ decreased as the RSI increased, which was likely due to the target color activation decaying over time. This decreased activation and influence of the trial $n-1$ target color should have a reduced the influence on target selection in trial $n$, but not necessarily reduced the need to retrieve prior instances of the target. Hence, this would result in smaller PoP effects on $\mu$ as RSI increased (due to reduced influence of the trial $n-1$ target on selection), but little change in the PoP effects on $\tau$ as RSI increased, which was observed. Thus, the results of the exGaussian analyses suggest that at least two processes were influenced by intertrial priming, consistent with Lamy et al. (2010) and Kristjánsson and Campana (2010).

There are limitations to using ex-Gaussian analyses to infer what processes may be operating, because equating exGaussian parameters with unique cognitive processes has been questioned for two main reasons (Balota \& Yap, 2011; Luce, 1986; Matzke \& Wagenmakers, 2009). First, studies have been inconsistent with regard to which parameter is associated with what process. Some studies liken $\mu$ to motor processes (Hohle, 1965), whereas others liken $\tau$ to motor processes (McGill, 1963; McGill \& Gibbon, 1965). As we mentioned above, there has been little discussion in the literature about what processes might contribute to changes in the $\sigma$ component, which is why we restricted our discussion above to the $\mu$ and $\tau$ components. However, recent studies, especially in the word recognition and lexical decision literatures, seem to agree that $\mu$ is sensitive to changes in stimulusdriven processes (e.g., selection, perception) and that $\tau$ is sensitive to slower-engaging and attention-demanding top-down processes (e.g., Balota \& Yap, 2011; Balota et al. 2008; Heathcote et al. 1991; Yap et al. 2006).

A second issue, articulated by Matzke and Wagenmakers (2009), is that "the ex-Gaussian distribution lacks a plausible theoretical basis" (p. 799), meaning the ex-Gaussian does not make a priori assumptions regarding which processes map onto its parameters. We agree this is a valid criticism of the ex-Gaussian analysis, and the results of the present study should be treated as descriptive and suggestive of multiple processes underlying intertrial priming. Matzke and Wagenmakers recommend diffusion model analyses of RTs (e.g., Ratcliff, 1978; Ratcliff \& McKoon, 2008; Wagenmakers, 2009), which combines RT and accuracy data to model performance, because the parameters of the models have well-defined cognitive interpretations. For example, the Ratcliff diffusion model assumes that RTs are the sum of decision time $(D T)$ and nondecision time $\left(T_{\mathrm{er}}\right)$ processes $(R T=$ $D T+T_{\text {er }}$; e.g., Ratcliff, 1978; Ratcliff \& McKoon, 2008; Ratcliff \& Rouder, 2000; Ratcliff, Thapar, \& McKoon, 2004; Wagenmakers, van der Maas \& Grasman, 2007). Although a full description of the Ratcliff model is beyond the scope of this study, the model assumes responding is influenced by several parameters (diffusion processes), each of which can have a theoretical connection to cognitive processes: (1)the mean drift rate $(v)$, which is the rate of evidence accumulation in favor of one response over another, or the "quality of information"; (2)intertrial variability in drift rate $(\eta)$; (3)response boundary separation $(a)$, which is one's conservativeness in responding; (4)starting point $(z)$, which is one's bias to favor one response over another, but is usually assumed to be halfway between two response boundaries; (5) variability in the starting point $\left(s_{\mathrm{z}}\right)$; (6)nondecision time $\left(T_{\mathrm{er}}\right)$, which is the total of all encoding and response processes; and (7) intertrial variability in the nondecision component $\left(s_{t}\right)$. Although the full model is complex, Wagenmakers et al. (2007); see also Grasman, Wagenmakers, \& van der Maas, 2009; Wagenmakers, van der Maas, Dolan, \& Grasman, 2008) proposed a simplified EZ-diffusion model, which incorporates the three most important parameters (drift rate, nondecision time, and boundary separation).

Diffusion models are relevant to this study and our discussion of "mapping" processes onto the ex-Gaussian parameters, because it is possible the changes to shift and skew observed in this study could be attributed to a single diffusion process. ${ }^{5}$ Stated differently, intertrial priming may have influenced a single diffusion process, which changed the scaling combined shift and skew - of the RT distributions between the repeat and reverse conditions. If so, a single diffusion

\footnotetext{
${ }^{5}$ I thank an anonymous reviewer for pointing this out.
} 
(cognitive) process could account for PoP effects. To examine this, using the data from Experiment 1 and the EZ-diffusion model equations (Wagenmakers et al. 2007), we calculated the drift rate $(v)$, boundary separation $(a)$, and nondecision time $\left(T_{\text {er }}\right)$ separately for the repeat and reverse conditions for each subject. Averages over the $n=15$ subjects are provided in Table 3, with the results of paired-samples $t$ tests that compared the repeat and reverse conditions. As seen in Table 3, intertrial priming influenced both drift rate and nondecision time; specifically, evidence accumulation ( $v$ ) was greater/ faster and nondecision time $\left(T_{\mathrm{er}}\right)$ was less in the repeat than in the reverse condition, suggesting two processes underlie PoP effects.

Additional research (planned and under way) will introduce other manipulations to influence selection or retrieval processes, to examine their influences on $M_{\mathrm{RT}}$ as well as on the ex-Gaussian parameters (or diffusion model parameters). For example, influencing target-distractor discriminability by manipulating target-distractor color/hue similarity should affect selection more than retrieval and have influences on different areas of the RT distributions. As an alternative approach, one could examine whether individual differences in working memory capacity covary with PoP effects on $M_{\mathrm{RT}}$ and on the ex-Gaussian parameters (Schmiedek, Oberauer, Wilhelm, Süß, \& Wittmann, 2007). Specifically, if working memory capacity reflects executive attention and the ability to control and retrieve information, one might predict that a measure of working memory capacity (e.g., OSPAN) would covary with PoP in the $\tau$ parameter (see Hutchison, Heap, Neely, \& Thomas, 2014, for an example with lexical priming). Lastly, varying the context of the displays should influence episodic retrieval and, potentially, influence only the $\tau$ parameter.

\section{Conclusion}

In this study, we examined the influences of intertrial priming on the shift, skew, and dispersion of the underlying RT distributions obtained in PoP tasks. All three experiments revealed that both the ex-Gaussian components $\mu$ and $\tau$ contributed to the PoP effects on $M_{\mathrm{RT}}$. More importantly, Experiments 2 and 3 demonstrated that two different manipulations (set size in Exp. 2 and RSI in Exp.3) produced changes in the sizes of the PoP effects on $\mu$ and $\tau$. Although it is questionable what specific cognitive processes might be reflected in ex-Gaussian parameters, the results of the study support Kristjánsson and Campana's (2010) argument that no single process can explain intertrial priming effects, and also support Lamy et al. (2010) dual-stage model, which assumes that two processes underlie intertrial priming's effects on visual search.

\section{References}

Andrews, S., \& Heathcote, A. (2001). Distinguishing common and taskspecific processes in word identification: A matter of some moment? Journal of Experimental Psychology: Learning, Memory, and Cognition, 27, 514-544. doi:10.1037/0278-7393.27.2.514

Balota, D. A., \& Spieler, D. H. (1999). Word frequency, repetition, and lexicality effects in word recognition tasks: Beyond measures of central tendency. Journal of Experimental Psychology: General, 128, 32-55. doi:10.1037/0096-3445.128.1.32

Balota, D. A., \& Yap, M. J. (2011). Moving beyond the mean in studies of mental chronometry: The power of response time distribution analyses. Current Directions in Psychological Science, 20, 160-166. doi:10.1177/0963721411408885

Balota, D. A., Yap, M. J., Cortese, M. J., \& Watson, J. M. (2008). Beyond mean response latency: Response time distributional analyses of semantic priming. Journal of Memory and Language, 59, 495523. doi:10.1016/j.jml.2007.10.004

Becker, S. I. (2008). The mechanism of priming: Episodic retrieval or priming of pop-out. Acta Psychologica, 127, 324-339. doi:10.1016/ j.actpsy.2007.07.005

Bichot, N. P., \& Schall, J. D. (2002). Priming in macaque frontal cortex during popout visual search: Feature based facilitation and locationbased inhibition of return. Journal of Neuroscience, 22, 4675-4685.

Burnham, B. R. (2013). Response retrieval in a go/no-go priming of popout task. Psychonomic Bulletin \& Review, 20, 1187-1194. doi: 10.3758/s13423-013-0433-0

Chun, M. M., \& Jiang, Y. (1998). Contextual cueing: Implicit learning and memory of visual context guides spatial attention. Cognitive Psychology, 36, 28-71. doi:10.1006/cogp.1998.0681

Chun, M. M., \& Nakayama, K. (2000). On the functional role of implicit visual memory for the adaptive deployment of attention across scenes. Visual Cognition, 7, 65-81. doi:10.1080/135062800394685

Cohen, J. (1988). Statistical power analysis for the behavioral sciences (2nd ed.). Hillsdale, NJ: Erlbaum.

Cumming, G. (2012). Understanding the new statistics: Effect sizes, confidence intervals, and meta-analysis. New York, NY: Routledge.

Franz, V. H., \& Loftus, G. R. (2012). Standard errors and confidence intervals in within-subjects designs: Generalizing Loftus and Masson (1994) and avoiding the biases of alternative accounts. Psychonomic Bulletin \& Review, 19, 395-404. doi:10.3758/ s13423-012-0230-1

Goolsby, B. A., \& Suzuki, S. (2001). Understanding priming of colorsingleton search: Roles of attention at encoding and "retrieval.". Perception \& Psychophysics, 63, 929-944. doi:10.3758/ BF03194513

Grasman, R. P. P. P., Wagenmakers, E.-J., \& van der Maas, H. L. J. (2009). On the mean and variance of response times under the diffusion model with an application to parameter estimation. Journal of Mathematical Psychology, 53, 55-68. doi:10.1016/j.jmp.2009.01. 006

Heathcote, A. (1996). RTSYS: A DOS application for the analysis of reaction time data. Behavior Research Methods, Instruments \& Computers, 28, 427-445. doi:10.3758/BF03200523

Heathcote, A. (2004). Fitting Wald and ex-Wald distributions to response time data: An example using functions for the S-plus package. Behavior Research Methods, Instruments, \& Computers, 36, 678694. doi:10.3758/BF03206550

Heathcote, A., Brown, S., \& Mewhort, D. J. K. (2002). Quantile maximum likelihood estimation of response time distributions. Psychonomic Bulletin \& Review, 9, 394-401. doi:10.3758/ BF03196299

Heathcote, A., Popiel, S. J., \& Mewhort, D. J. K. (1991). Analysis of response time distributions: An example using the Stroop task. 
Psychological Bulletin, 109, 340-347. doi:10.1037/0033-2909.109. 2.340

Hillstrom, A. P. (2000). Repetition effects in visual search. Perception \& Psychophysics, 62, 800-817. doi:10.3758/BF03206924

Hockley, W. E. (1984). Analysis of response time distributions in the study of cognitive processes. Journal of Experimental Psychology: Learning, Memory and Cognition, 10, 598-615. doi:10.1037/02787393.10.4.598

Hohle, R. H. (1965). Inferred components of reaction times as functions of foreperiod duration. Journal of Experimental Psychology, 69, 382-386. doi:10.1037/h0021740

Hollands, J. G., \& Jarmasz, J. (2010). Revisiting confidence intervals for repeated measures designs. Psychonomic Bulletin \& Review, 17, 135-138. doi:10.3758/PBR.17.1.135

Huang, L., Holcombe, A. O., \& Pashler, H. (2004). Repetition priming in visual search: Episodic retrieval, not feature priming. Memory \& Cognition, 32, 12-20. doi:10.3758/BF03195816

Huang, L., \& Pashler, H. (2005). Expectation and repetition effects in searching for featural singletons in very brief displays. Perception \& Psychophysics, 67, 150-157. doi:10.3758/BF03195018

Hutchison, K. A., Heap, S. J., Neely, J. H., \& Thomas, M. A. (2014). Attentional control and asymmetric associative priming. Journal of Experimental Psychology: Learning, Memory, and Cognition, 40, 844-856. doi:10.1037/a0035781

Kristjánsson, Á., \& Campana, G. (2010). Where perception meets memory: A review of repetition priming in visual search tasks. Attention, Perception, \& Psychophysics, 72, 5-18. doi:10.3758/APP.72.1.5

Kristjánsson, Á., \& Jóhannesson, Ó. I. (2014). How priming in visual search affects response time distributions: Analyses with exGaussian fits. Attention, Perception, \& Psychophysics, 76, 21992211. doi:10.3758/s13414-014-0735-y

Kristjánsson, Á., Wang, D., \& Nakayama, K. (2002). The role of priming in conjunctive visual search. Cognition, 85, 37-52. doi:10.1016/ S0010-0277(02)00074-4

Lamy, D., Antebi, C., Aviani, N., \& Carmel, T. (2008). Priming of popout provides reliable measures of target activation and distractor inhibition in selective attention. Vision Research, 48, 30-41.

Lamy, D., Carmel, T., Egeth, H. E., \& Leber, A. B. (2006). Effects of search mode and intertrial priming on singleton search. Perception \& Psychophysics, 68, 919-932. doi:10.3758/BF03193355

Lamy, D., Yashar, A., \& Ruderman, L. (2010). A dual-stage account of intertrial priming effects. Vision Research, 50, 1396-1401. doi:10. 1016/j.visres.2010.01.008

Luce, R. D. (1986). Response times: Their role in inferring elementary mental organization. New York, NY: Oxford University Press.

Maljkovic, V., \& Nakayama, K. (1994). Priming of pop-out: I. Role of features. Memory \& Cognition, 22, 657-672. doi:10.3758/ BF03209251

Maljkovic, V., \& Nakayama, K. (1996). Priming of pop-out: II. The role of position. Perception \& Psychophysics, 58, 977-991. doi:10.3758/ BF03206826

Maljkovic, V., \& Nakayama, K. (2000). Priming of pop-out: III. A shortterm implicit memory system beneficial for rapid target selection. Visual Cognition, 7, 571-595. doi:10.1080/135062800407202

Matzke, D., \& Wagenmakers, E.-J. (2009). Psychological interpretation of the ex-Gaussian and shifted Wald parameters: A diffusion model analysis. Psychonomic Bulletin \& Review, 16, 798-817. doi:10. 3758/PBR.16.5.798

McGill, W. J. (1963). Stochastic latency mechanisms. In R. D. Luce, R. R. Bush, \& E. Galanter (Eds.), Handbook of mathematical psychology (Vol. 1, pp. 309-360). New York, NY: Wiley.

McGill, W. J., \& Gibbon, J. (1965). The general-gamma distribution and reaction times. Journal of Mathematical Psychology, 2, 1-18.

Meeter, M., \& Olivers, C. N. L. (2006). Intertrial repetition stemming from ambiguity: A new account of priming in visual search. Visual Cognition, 13, 202-222.
Müller, H. J., Heller, D., \& Ziegler, J. (1995). Visual search for singleton feature targets within and across feature dimensions. Perception \& Psychophysics, 57, 1-17. doi:10.3758/BF03211845

Olivers, C. N. L., \& Humphreys, G. W. (2003). Attentional guidance by salient feature singletons depends on intertrial contingencies. Journal of Experimental Psychology: Human Perception and Performance, 29, 650-657. doi:10.1037/0096-1523.29.3.650

Palmer, E. M., Horowitz, T. S., Torralba, A., \& Wolfe, J. M. (2011). What are the shapes of response time distributions in visual search? Journal of Experimental Psychology: Human Perception and Performance, 37, 58-71. doi:10.1037/a0020747

Ratcliff, R. (1978). A theory of memory retrieval. Psychological Review, 85, 59-108. doi:10.1037/0033-295X.85.2.59

Ratcliff, R., \& McKoon, G. (2008). The diffusion decision model: Theory and data for two-choice decision tasks. Neural Computation, 20, 873-922. doi:10.1162/neco.2008.12-06-420

Ratcliff, R., \& Rouder, J. N. (2000). A diffusion model account of masking in two-choice letter identification. Journal of Experimental Psychology: Human Perception and Performance, 26, 127-140. doi:10.1037/0096-1523.26.1.127

Ratcliff, R., Thapar, A., \& McKoon, G. (2004). A diffusion model analysis of the effects of aging on recognition memory. Journal of Memory and Language, 50, 408-424. doi:10.1016/j.jml.2003.11. 002

Rouder, J. N. (2005). Are unshifted distributional models appropriate for response time? Psychometrika, 70, 377-381.

Rouder, J. N., \& Speckman, P. L. (2004). An evaluation of the Vincentizing method for forming group-level response time distributions. Psychonomic Bulletin \& Review, 11, 419-427. doi:10.3758/ BF03196589

Schmiedek, F., Oberauer, K., Wilhelm, O., Süß, H.-M., \& Wittmann, W. W. (2007). Individual differences in components of reaction time distributions and their relations to working memory and intelligence. Journal of Experimental Psychology: General, 136, 414-429. doi: 10.1037/0096-3445.136.3.414

Schwarz, W. (2001). The ex-Wald distribution as a descriptive model of response times. Behavior Research Methods, Instruments, \& Computers, 33, 457-469. doi:10.3758/BF03195403

Thomson, D. R., \& Milliken, B. (2011). A switch in task affects priming of pop-out: Evidence for the role of episodes. Attention, Perception \& Psychophysics, 73, 318-333. doi:10.3758/s13414-010-0046-x

Thomson, D. R., \& Milliken, B. (2012). Perceptual distinctiveness produces long-lasting priming of pop-out. Psychonomic Bulletin \& Review, 19, 170-176. doi:10.3758/s13423-011-0199-1

Thomson, D. R., \& Milliken, B. (2013). Contextual distinctiveness produces long-lasting priming of pop-out. Journal of Experimental Psychology. Human Perception and Performance, 39, 202-215. doi:10.1037/a0028069

Tse, C.-S., Hutchison, K. A., \& Li, Y. (2011). Effects of contextual similarity and target-repetition proportion on negative priming in RT distribution analyses. Journal of Experimental Psychology: Human Perception and Performance, 37, 180-192.

Van Zandt, T. (2000). How to fit a response time distribution. Psychonomic Bulletin \& Review, 19, 424-465. doi:10.3758/ BF03214357

Wagenmakers, E.-J. (2009). Methodological and empirical developments for the Ratcliff diffusion model of response times and accuracy. European Journal of Cognitive Psychology, 21, 641-671. doi:10. 1080/09541440802205067

Wagenmakers, E.-J., van der Maas, H. L. J., Dolan, C. V., \& Grasman, R. P. P. P. (2008). EZ does it! Extensions of the EZ-diffusion model. Psychonomic Bulletin \& Review, 15, 1229-1235. doi:10.3758/PBR. 15.6.1229

Wagenmakers, E.-J., van der Maas, H. L. J., \& Grasman, R. P. P. P. (2007). An EZ-diffusion model for response time and accuracy. 
Psychonomic Bulletin \& Review, 14, 3-22. doi:10.3758/ BF03194023

Wenger, M. J., \& Gibson, B. S. (2004). Using hazard functions to assess changes in processing capacity in an attentional cuing paradigm. Journal of Experimental Psychology: Human Perception and Performance, 30, 708-719. doi:10.1037/00961523.30.4.708

Wolfe, J. M., Butcher, S. J., Lee, C., \& Hyle, M. (2003). Changing your mind: On the contributions of top-down and bottom-up guidance in visual search for feature singletons. Journal of Experimental Psychology: Human Perception and Performance, 29, 483-502. doi:10.1037/0096-1523.29.2.483
Yap, M. J., Balota, D. A., Cortese, M. J., \& Watson, J. M. (2006). Singleversus dual-process models of lexical decision performance: Insights from response time distribution analysis. Journal of Experimental Psychology: Human Perception and Performance, 32, 1324-1344. doi:10.1037/0096-1523.32.6.1324

Yap, M. J., \& Seow, C. S. (2014). The influence of emotion on lexical processing: Insights from RT distributional analysis. Psychonomic Bulletin \& Review, 21, 526-533. doi:10.3758/s13423-013-0525-x

Yashar, A., \& Lamy, D. (2011). Refining the dual-stage account of intertrial feature priming: Does motor response or response feature matter? Attention, Perception, \& Psychophysics, 73, 2160-2167. doi: 10.3758/s13414-011-0182-y 